\title{
СРЕДСТВА ПОВЫШЕНИЯ ЭФФЕКТИВНОСТИ СИСТЕМЫ БЕСКОНТАКТНОГО ЗАРЯДА АККУМУЛЯТОРНЫХ БАТАРЕЙ АВТОНОМНОГО ПОДВОДНОГО АППАРАТА
}

\section{В.А. Герасимов, А.В. Комлев, А.Ю. Филоженко}

Федеральное государственное бюджетное учреждение науки Институт проблем морских технологий ДВО РАН ${ }^{1}$

Необходимым условием эффективности системы бесконтактного заряда аккумуляторных батарей при эксплуатации автономного подводного робота служит выполнение следующих двух требований:

- сочетание резонансного контура на выходе инвертора и режима «мягкого переключения» силовых ключей инвертора,

- применение резонанса на вторичной стороне трансформатора.

Для решения задачи, связанной с данным условием, разработана математическая модель системы с рекомендациями по выбору параметров резонансных цепей. Таким путем достигаются: минимум потерь мощности в инверторе, а также уменьшение нагрева его элементов и, как следствие, повышение общей надежности и минимум времени полного заряда аккумуляторных батарей. С помощью действующего образца системы проведены натурные эксперименты, подтверждающие результаты исследований.

\section{ВВЕДЕНИЕ}

Источником энергии для функционирования автономного необитаемого подводного аппарата (АНПА) в большинстве его применений являются аккумуляторные батареи, зарядка которых может выполняться различными способами [1-4]. Самый простой способ по аппаратной реализации, но технологически наиболее трудоемкий заключается в подъеме аппарата на борт обеспечивающего судна, выполнении необходимых монтажных и коммутационных процедур и осуществлении заряда батарей от какого-либо зарядного устройства.

Повышение оперативности этого процесса со значительным расширением функциональных возможностей применения АНПА достигается при выполнении зарядки его батарей с использованием бесконтактного способа передачи электроэнергии. В таком процессе участвует специальный трансформатор с разделяющимися первичной и вторичной частями, которые представляют собой прочные герметичные оболочки с размещенными внутри электрическими обмотками определенной конфигурации. Первичная часть такого трансформатора помещается на базе, а вторичная устанавливается на АНПА. Для питания первичной обмотки трансформатора при- меняется автономный инвертор напряжения (АИН), который выполняет преобразование электроэнергии базового источника в напряжение высокой частоты, способствующее снижению массы и габаритов трансформатора, а также обеспечивает необходимое регулирование передаваемой электроэнергии [4].

В качестве базы могут использоваться различные подводные и береговые объекты. Это может быть, например, так называемый «гараж», опускаемый с обеспечивающего судна под воду с помощью специальных спускоподъемных устройств. В этом случае передача электроэнергии к АИН, расположенному совместно с первичной частью трансформатора в «гараже», осуществляется по кабель-тросу, который дополнительно к электрической связи обеспечивает как механическую силовую связь между судном и «гаражом», так и функционирование информационного канала между оператором на обеспечивающем судне и АНПА.

Некоторые задачи применения АНПА требуют организации его длительного подводного базирования, при этом конструкция базы причаливания для АНПА выполняется в виде донного причального устройства (ДПУ), устанавливаемого под водой на грунте ста-

${ }^{1}$ 690091, г. Владивосток, ул. Суханова, 5а. Тел.: +7 (423) 243-25-78. E-mail: gerasimov@marine.febras.ru 
ционарно. Электроснабжение ДПУ может осуществляться с использованием кабеля, связывающий его ДПУ с береговым центром управления БЦУ, при этом наряду с передачей электроэнергии одновременно организуется также информационный канал [5].

Обеспечение длительного базирования подводного аппарата на ДПУ актуально при неизменном районе действия, например, при охране порта или мониторинге подводной обстановки заданного района. Применение этой технологии в военных целях позволяет решить проблему автономности и существенно увеличить время нахождения аппарата на позиции. Использование бесконтактной передачи электроэнергии для заряда аккумуляторных батарей АНПА под водой в автоматическом режиме в случае его подводного базирования практически не имеет альтернативы [5].

Размещение функциональных элементов системы электроснабжения АНПА и схема связи между ними при использовании ДПУ показаны на рис. 1.

Составными элементами системы бесконтактного заряда являются автономный инвертор напряжения АИН, высокочастотный трансформатор с разделенными первичной Т1 и вторичной Т2 частями, автоматическое зарядное устройство АЗУ и аккумуляторная батарея АБ. Первичная Т1 и вторичная Т2 части представляют собой отдельные герметичные оболочки, в которые помещены обмотки трансформатора. В режиме передачи энергии оси обмоток должны совпадать, а их торцы находиться на минимальном расстоянии друг относительно друга. Каждая из оболочек имеет выполненную из изоляционного материала контактную стенку, толщина которой достигает нескольких миллиметров. В связи с наличием этого немагнитного конструктивного зазора между торцами обмоток такой трансформатор имеет низкое значение коэффициента магнитной связи между обмотками [6].

Это вызывает увеличение значения намагничивающей составляющей входного тока трансформатора,

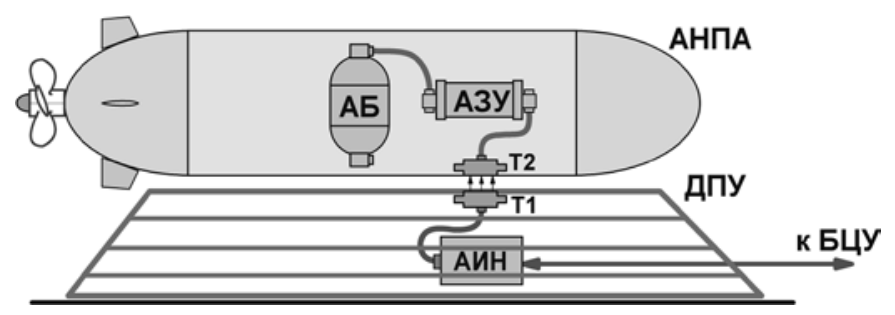

Puc. 1. Функциональная схема системы бесконтактной передачи электроэнергии для заряда аккумуляторных батарей при базировании АНПА на ДПУ: АБ - аккумуляторная батарея, АЗУ - автоматическое зарядное устройство, АИН - автономный инвертор напряжения; Т1, Т2 - первичная и вторичная части высокочастотного трансформатора что приводит к уменьшению коэффициента мощности АИН и эквивалентному увеличению выходного тока инвертора при передаче определенного значения активной мощности. При этом выходной ток АИН увеличивается в несколько раз по сравнению с минимально необходимым значением, определяемым нагрузкой трансформатора на его вторичной стороне. В общем случае это обстоятельство вызывает такие последствия, как необходимость выбора транзисторов и диодов АИН с завышенными номинальными токами; повышение потерь мощности в этих полупроводниковых приборах, увеличение их массы и стоимости; усложнение проблемы отвода тепла, вызванного дополнительными потерями мощности.

Негативный характер этих последствий усиливается в системе энергообеспечения подводного аппарата, поскольку электронные блоки должны размещаться в герметичных контейнерах с плотной компоновкой и с ограниченными возможностями отвода тепла от силовых приборов.

Указанные обстоятельства определяют актуальность поиска решений, которые позволят снизить токовую нагрузку на силовые ключи АИН при сохранении заданного значения передаваемой активной мощности. В конечном итоге это должно привести к повышению надежности устройства и увеличению эффективности системы бесконтактного заряда АБ подводного аппарата в целом.

\section{1. Анализ работы автономного инвертора при холостом ходе на вторичной стороне трансформатора}

Решение поставленной задачи повышения эффективности рассматриваемой системы можно получить, применяя резонансные цепи как на первичной стороне трансформатора, так и на вторичной стороне, в сочетании с режимом мягкой коммутации. Реализация таких цепей РЦ1 и РЦ2, показанных на рис. 2, может быть различнаой, но итоговый результат повышения эффективности должен определяться суммой вносимых эффектов без взаимного влияния, что будет способствовать адекватному воспроизведению результатов расчета резонансных элементов при их практической реализации и упрощению общей настройки системы.

В общем случае суммарные потери мощности на ключах инвертора складываются из динамических потерь включения и выключения, а также статических потерь в открытом состоянии ключа. Потери от токов утечки в закрытом состоянии малы, и их можно не учитывать. В [7] основное внимание уделяется 


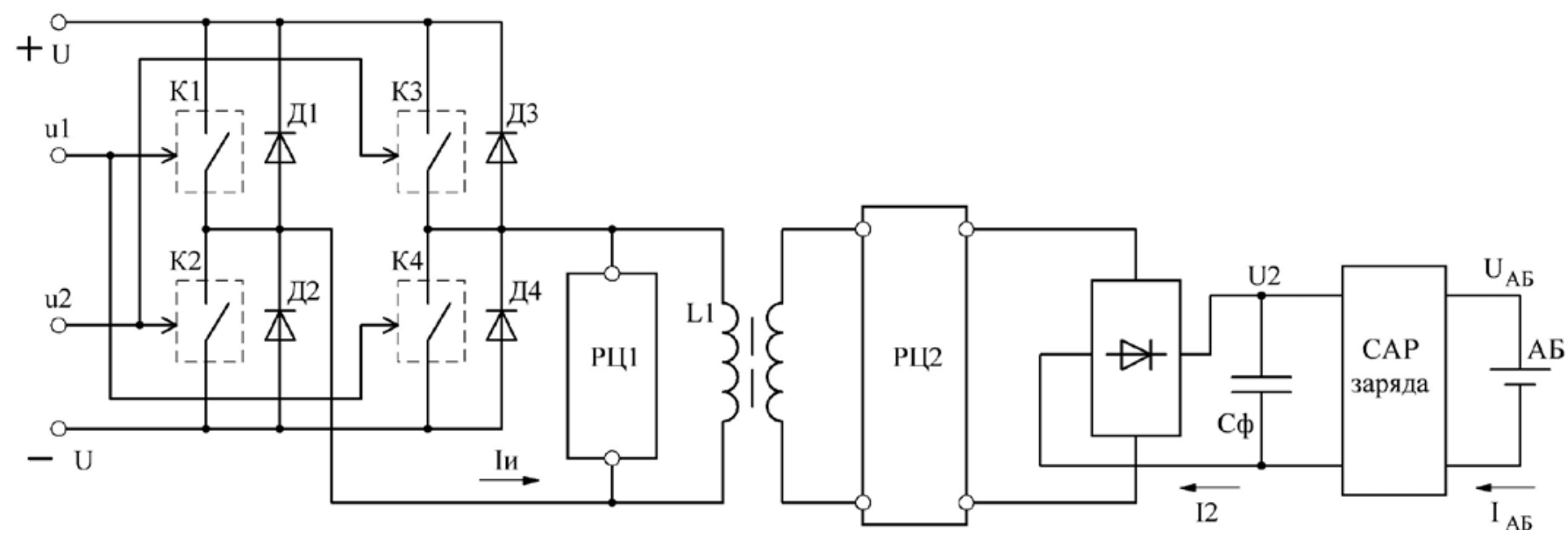

Puc. 2. Структурная схема системы бесконтактного заряда АБ

минимизации динамических потерь при включении, что достигается созданием условий включения при нулевом напряжении на ключе - режимом ZVS (zero voltage switching). Такой режим носит название «мягкой коммутации» при включении. Вместе с тем расчеты и эксперименты показывают, что как динамические потери при выключении, так и потери мощности на ключах в открытом состоянии также вносят значительный вклад в общую сумму потерь. Таким образом, можно поставить актуальную задачу обеспечения условий «мягкой коммутации» при выключении, что достигается, очевидно, выключением при нулевом токе - режимом ZCS (zero current switching). Одновременно с этим актуальной является задача снижения потерь проводимости в интервале открытого состояния ключей. Отметим, что при любых вариантах корректирующих воздействий на систему должно сохраняться неизменным значение передаваемой активной мощности.

Решение задачи токовой разгрузки ключей в статическом режиме открытого состояния можно искать, очевидно, в виде такой структуры РЦ1, которая, будучи подключена параллельно первичной обмотке с индуктивностью L1, осуществляет компенсацию намагничивающей составляющей входного тока трансформатора. Поскольку эта составляющая имеет индуктивный характер, то компенсацию следует выполнять некоторым элементом, имеющим емкостной характер нагрузки. Применение конденсатора в чистом виде здесь ограничено, т.к. на выходе инвертора формируется напряжение прямоугольной формы.

Одним из известных способов токовой разгрузки силовых ключей в статическом режиме является включение Г-образного $L C$-фильтра низких частот на выходе инвертора. При этом нагрузка инвертора (первичная обмотка трансформатора) подключается параллельно конденсатору фильтра [8--11]. Такое решение позволяет разгрузить ключи АИН, однако из-за падения напряжения на реакторе искажается внешняя характеристика трансформатора и, как следствие, снижается передаваемая мощность.

Лучшие результаты получаются при использовании последовательной резонансной $L C$-цепи, подключаемой на выходе инвертора параллельно первичной обмотке трансформатора [9]. Эта цепь так же компенсирует индуктивную составляющую выходного тока инвертора за счет своего емкостного характера, но практически не влияет на форму и значения токов обмоток трансформатора. Это обеспечивает снижение действующего значения выходного тока инвертора при сохранении уровня передаваемой активной мощности. Применяемая резонансная цепь имеет собственную резонансную частоту $f_{I U}$, соответствующую резонансу напряжений [10]:

$$
f_{1 U}=\frac{1}{2 \pi \sqrt{L_{P} \cdot C_{P 1}}},
$$

где $L_{P}$ и $C_{P I}-$ соответственно индуктивность и емкость резонансной цепи на первичной стороне трансформатора.

Вместе с тем рабочая частота $f$ коммутации АИН должна соответствовать частоте $f_{I I}$ резонанса токов в нагрузке АИН, состоящей из параллельно включенных резонансной последовательной $L C$-цепи и первичной обмотки трансформатора $L_{l}$. При этом условии обеспечивается минимальная токовая нагрузка на транзисторные ключи АИН. Для первой гармоники переменного напряжения на выходе АИН справедливо соотношение:

$$
f=f_{1 I}=\frac{1}{2 \pi \sqrt{\left(L_{P}+L_{1}\right) C_{P 1}}},
$$

где $L_{1}-$ индуктивность первичной обмотки трансформатора. 
Если ограничить условия анализа работы АИН первой гармоникой его выходных колебаний, то индуктивность $L_{P}$ реактора и емкость $C_{P I}$ конденсатора последовательной резонансной цепи РЦ1 можно определить по формулам [10]:

$$
\begin{gathered}
L_{P}=\frac{L_{1}}{m^{2}-1}, \\
C_{P 1}=\frac{m^{2}-1}{(2 \pi \cdot m \cdot f)^{2} L_{1}},
\end{gathered}
$$

где параметр $m=f_{1 U} / f_{1 I}$ - это отношение собственной частоты резонансной $L C$-цепи (частоты резонанса напряжений) к рабочей частоте коммутации АИН (частоте резонанса токов цепи нагрузки АИН). Выбор численного значения параметра $m$ определяется на основе компромисса между желаемым снижением тока ключей АИН и приемлемыми значениями реактивных мощностей емкости и индуктивности резонансной цепи, что обусловливает массу и габариты этих элементов.

Для получения резонанса токов в нагрузке АИН для первой гармоники его выходного напряжения значение относительной частоты $n_{1 I}=f / f_{1 I}$ должно быть равно единице. С учетом того, что на выходе АИН формируется прямоугольный сигнал, для получения минимального действующего значения выходного тока АИН коэффициент $n_{11}$ должен быть несколько больше единицы. Это значение относительной частоты $n_{1 l}$ было установлено путем математического моделирования в программе MatLab с использованием моделей, приведенных в $[10,12]$.

Для анализа полученных временных диаграмм тока $I_{H}(t)$ инвертора примем нумерацию ключей и оппозитных диодов в составе силовой части АИН, как показано на рис. 2. Результаты моделирования для режима холостого хода трансформатора, иллюстрирующие эффект от введения резонансной $L C$-цепи РЦ1, приведены на рис. 3.

Сигналы управления $u_{1}$ и $u_{2}$ инвертором поступают на диагонально расположенные ключи и имеют интервал «dead time» при переключении, что предотвращает аварийные сквозные токи через ключи одной стойки $K_{1}, K_{2}$ или $K_{3}, K_{4}$. Графики на рисунке $3, a$ соответствуют состоянию, когда резонансная $L C$ цепь отсутствует и к выходу АИН подключена только первичная обмотка трансформатора с собственной индуктивностью $L_{l}$. В этом случае ток в нагрузке изменяется линейно и протекает поочередно через диагональные ключи или через диоды. На рис. $3, a$ показаны интервалы проводимости ключей (двойная линия) и диодов (пунктир) для этого случая на
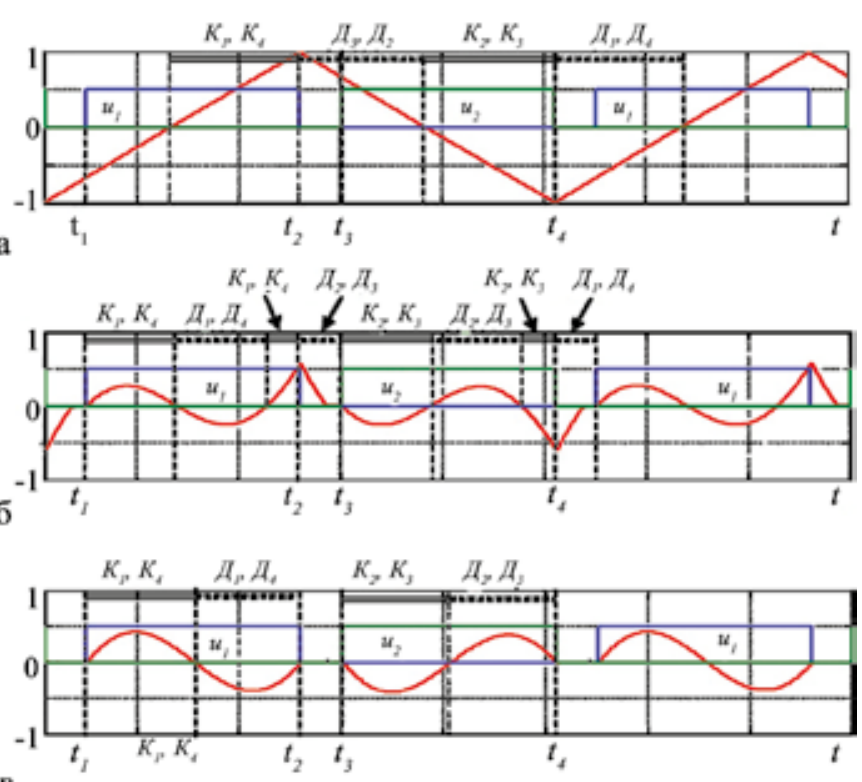

B

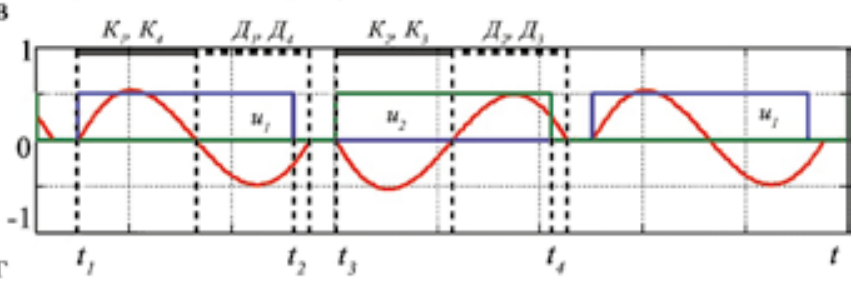

Puc. 3. Диаграммы выходного тока АИН при изменении параметров резонансной цепи: a - резонансная цепь отсутствует; $\sigma$ параметры резонансной цепи соответствуют относительной частоте $n_{11}=1 ; 8,2$ - параметры резонансной цепи соответствуют нулевым потерям переключения при $n_{11}>1$

протяжении одного периода коммутации. Управляющий сигнал $u_{1}$ воздействует на входы ключей $K_{1}, K_{4}$ в интервале времени $t_{1} \ldots t_{2}$, затем следует пропуск «dead time» в интервале $t_{2} \ldots t_{3}$ и далее управление $u_{2}$ поступает на ключи $K_{2}, K_{3}$ второй диагонали моста инвертора в интервале времени $t_{3} \ldots t_{4}$. Интервалы проводимости ключей и диодов чередуются, при этом ключи каждой диагонали проводят ток при воздействии соответствующего управляющего сигнала $u_{1}$ или $u_{2}$ после завершения интервала проводимости диодов.

Если за единичное значение принять ток инвертора в моменты закрывания ключей для работы без резонансной цепи, то действующее значение тока инвертора для рассматриваемого случая (треугольной формы тока) будет равно $1 / \sqrt{3}$, что по отношению к режиму с резонансом является повышенным. Кроме этого, как следует из рис. $3, a$, в моменты времени закрывания ключей (момент $t_{2}$ для $K_{1}, K_{4}$ и момент $t_{4}$ для $K_{2}, K_{3}$ ) ток через ключи имеет максимальное значение. Это приводит к значительным потерям мощности на ключах в режиме выключения, проблемам отвода тепла и увеличенному нагреву силовых элементов инвертора. 
При подключении параллельно первичной обмотке трансформатора последовательной резонансной $L C$-цепи РЦ1 форма выходного тока инвертора и интервалы проводимости ключей и диодов изменяются. Для первой гармоники выходного напряжения АИН условие $n_{1 I}=f / f_{1 I}>1$ соответствует резонансу токов в его нагрузке. При этом действующее значение выходного тока инвертора имеет минимальное значение, определяемое активными потерями в цепях протекания тока. Для реального выходного сигнала инвертора в виде меандра минимальное действующее значение тока инвертора достигается при $n_{1 I}=f /$ $f_{1 I}>1$. Форма выходного тока инвертора и интервалы проводимости ключей и диодов будут иметь вид, как показано на рис. 3,6 .

Результаты моделирования показывают, что таким образом можно уменьшить действующее значение тока инвертора примерно в 2,5 раза $[10,11]$. Однако соответствующего уменьшения потерь мощности здесь не происходит. Причиной такого результата является форма тока инвертора, при которой так же, как и в исходном варианте, в моменты времени $t_{2}$ и $t_{4}$ выключения ключей ток имеет существенное значение. Это значение меньше, чем амплитуда пилообразной формы тока на рис. $3, a$, и равно примерно 0,6 , но этого оказывается достаточно, чтобы суммарные потери мощности в инверторе остались на высоком уровне.

Значительное снижение мощности потерь в инверторе можно получить при переходе к «мягкой коммутации», что будет выполняться при определенном значении относительной частоты $n_{1 I}>1$ (рис. 3, ) ). В этом режиме выключение транзисторов инвертора будет происходить при нулевом токе и сопровождаться минимальными динамическими потерями. Увеличение относительной частоты резонанса токов $n_{1 I}$ будет деформировать кривую выходного тока инвертора, как показано на рис. 3 , 2, и режим мягкой коммутации сохранится при увеличении интервала проводимости диодов $Д_{1}, Д_{4}$ до момента открытия ключей $K_{2}, K_{3}$, т.е. в интервале «мертвого времени». Дальнейшее повышение значения $n_{1 I}$ приведет к возрастанию потерь проводимости в открытом состоянии от увеличенной емкостной составляющей тока нагрузки инвертора, обусловленной РЦ1. Здесь нумерация диодов и ключей приведена для одного полупериода выходного сигнала инвертора. Для второго полупериода, в соответствии с рис. 2, в рассуждениях будут участвовать диоды $Д_{2}, Д_{3}$ и ключи $K_{1}, K_{4}$.

Таким образом, здесь возникает компромиссная задача определения значения относительной частоты $n_{1 p}$, приводящая к минимизации потерь в АИН как в режиме холостого хода трансформатора, так и в полном диапазоне его нагрузок, включая последствия от неточной стыковки передающей Т1 и приемной Т2 частей трансформатора при причаливании АНПА к базе. При этом минимизации должны подлежать как потери переключения, так и потери проводимости.

Для решения этой задачи при математическом моделировании АИН были получены зависимости тока $I_{O F F}{ }^{*}$ через транзисторы инвертора в момент подачи управляющего сигнала на отключение, а также действующее значения тока $I_{И}{ }^{*}$ инвертора и мощность $P_{n}{ }^{*}$ потерь в инверторе в функции относительной частоты $n_{1 I}=f / f_{1 \Gamma}$. Полученные результаты приведены на рис. 4 , где переменные показаны в относительных единицах. За единицу приняты значения этих переменных при отсутствии резонансной цепи РЦ1.

Из этих графиков следует, что значение тока $I_{O F F}{ }^{*}$ и, соответственно, потери при выключении достигают нуля при относительной частоте $n_{1 I}^{(a)}=1,09$. Область на плоскости графиков, расположенная справа от частоты $n_{1 I}^{(a)}$, соответствует области ZCS (переключение при нулевом токе).

Здесь нулевые потери на выключение сохраняются. Слева от частоты $n_{1 I}^{(b)}=1,19$ расположена область ZVS (переключение при нулевом напряжении), которая соответствует индуктивному режиму нагрузки инвертора и характеризуется нулевыми потерями на включение.

Таким образом, диапазон значений относительной частоты от $n_{1 I}^{(a)}=1,09$ до $n_{1 I}^{(b)}=1,19$, полученный при математическом моделировании соответствует окончанию интервала проводимости диодов $Д_{1}, Д_{4}$ (или диодов $Д_{2}, Д_{3}$ ) в диапазоне интервала «мертвого времени» (рис. 3,2$)$ и обеспечивает низкие потери мощности как на включение, так и на отключение транзисторов инвертора. При этом целесообразно принять значение относительной частоты из это-

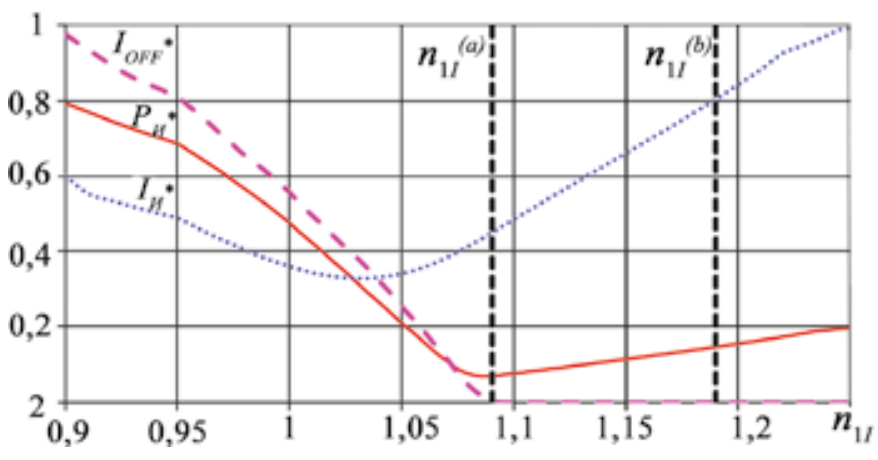

Puc. 4. Частотные характеристики АИН в режиме холостого хода: $I_{\text {OFF }}{ }^{*}$ - ток через ключи к моменту выключения; $P_{n}{ }^{*}-$ мощность потерь в АИН; $I_{n}{ }^{*}$ - действующее значение выходного тока АИН 
го диапазона ближе к левой его границе, например, $n_{1 I}=1,1$. Так суммарные потери на транзисторах будут минимальны. Следует учитывать, что при выборе значения относительной частоты потери переключения остаются нулевыми, а несколько увеличиваются потери проводимости за счет увеличения выходного тока инвертора.

Графики на рис. 4 определены для некоторого $m=f_{1 U} / f_{1 I}=$ const и соответствуют определенным значениям индуктивности реактора $L_{P}$ и емкости $C_{I P}$ резонансной цепи РЦ1. Вместе с тем представляет практический интерес определение такого значения параметра $m$, при котором мощности этих элементов будут минимальны при сохранении минимальных потерь в инверторе. Для этого можно использовать зависимости относительных мощностей $S_{L}^{*}, S_{C}^{*}$ реактора и емкости резонансной цепи РЦ1 соответственно в функции параметра $m$, которые определяются путем математического моделирования инвертора при условии обеспечения в нем минимальных потерь. Указанные величины определяются в отношении к мощности инвертора без резонансной цепи РЦ1.

На рис. 5 приведены графики относительных мощностей $S_{L}^{*}, S_{C}^{*}$ в функции параметра $m$, а также зависимости относительной мощности $S_{И}^{*}$ инвертора и относительных потерь $P_{И}^{*}$ в инверторе при подключении резонансной цепи РЦ1, которые также находятся в отношении к мощности потерь в инверторе без резонансной цепи.

Из рис. 5 следует, что мощность и, соответственно, габариты индуктивности и емкости достигают минимальных значений при $2<m<2,2$. Принимая параметр $m$ в этом диапазоне (например, $m=2$ ), можно определить требуемые значения $L_{P}$ и $C_{P I}$ резонансной цепи.

Отметим, что приведенные рассуждения относятся к холостому ходу трансформатора и подключение

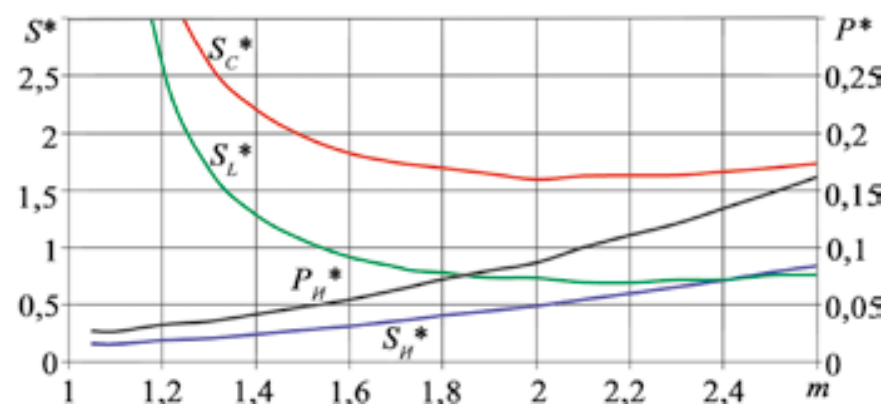

Puc. 5. Зависимости относительной мощности АИН и элементов резонансной $L C$-цепи от параметра $m$ при холостом ходе: $S_{n}{ }^{*}-$ выходная мощность АИН; $P_{h}^{*}-$ мощность потерь в АИН; $S_{C}^{*}, S_{L}^{*}-$ мощности конденсатора и реактора соответственно нагрузки не изменяет режим работы его первичной стороны.

\section{2. Характеристики системы бесконтактной передачи электроэнергии в режиме нагрузки на вторичной стороне}

При подключении к выводам вторичной обмотки трансформатора нагрузки параметры передаваемой в нее электроэнергии можно определить в виде внешних характеристик системы $U_{2}\left(I_{2}\right)$, где напряжение $U_{2}$ и ток $I_{2}$ показаны на рис. 2. Нагрузка в рассматриваемом случае представляет собой сочетание выпрямителя, фильтра $\mathrm{C}_{\Phi}$, импульсного преобразователя с системой автоматического регулирования (САР заряда) и аккумуляторной батареи АБ. Полный заряд батареи будет возможен, если к входу САР заряда подводится напряжение, значение которого превышает напряжение полностью заряженной батареи. Широкие возможности деформации исходной внешней характеристики системы в желаемом направлении представляются при введении на вторичной стороне трансформатора резонансной цепи РЦ2. Из возможных схемных реализаций представляет практический интерес выполнение РЦ2 в виде последовательного с вторичной обмоткой трансформатора конденсатора $C_{P 2}$. В определенных условиях может быть эффективно включение резонансного конденсатора $C_{P 2}$ параллельно вторичной обмотке трансформатора.

При включении последовательно с первичной обмоткой трансформатора конденсатора $C_{P 2}$ внешняя характеристика $U_{2}\left(I_{2}\right)$ системы передачи электроэнергии может иметь вид одной из характеристик, приведенных на рис. 6 , где $U_{2}^{*}$ - напряжение на входе САР заряда, а $I_{2}{ }^{*}$ - входной ток САР заряда АБ в относительных единицах, при этом напряжение холостого хода и ток короткого замыкания для этого графика

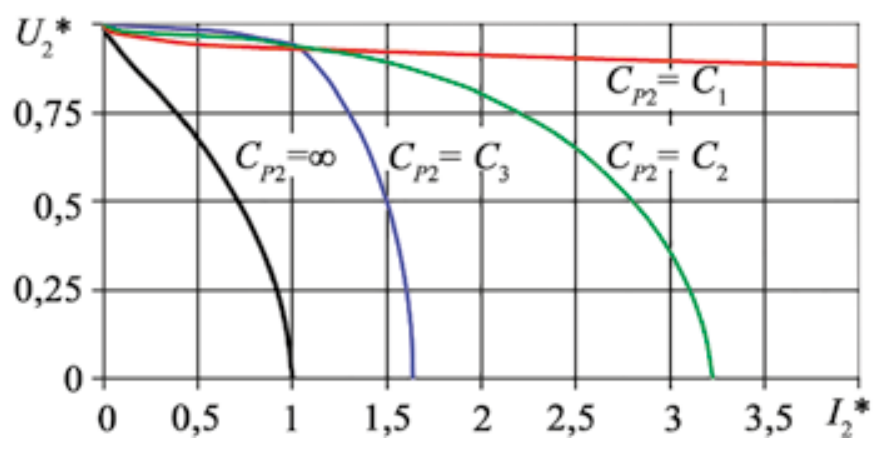

Puc. 6. Внешние характеристики системы передачи электроэнергии для различных значений резонансного конденсатора $C_{P}$ в цепи нагрузки: $C_{P 2}=C_{1}<C_{2}<C_{3}$ 
приняты за единичные базовые значения. Показанное семейство характеристик получается при изменении значения резонансного конденсатора $C_{P 2}[13,14]$.

Включение во вторичную цепь резонансного конденсатора вызывает деформацию внешних характеристик системы, как показано на рис. 6, при этом значение емкости $C_{P 2}=C_{1}$ соответствует резонансу напряжений. Уменьшение емкости относительно этого значения увеличивает статизм внешних характеристик. Практическое применение имеют характеристики в диапазоне изменения емкости резонансного конденсатора $C_{2}<C_{P 2}<C_{3}$. Можно считать, что внешняя характеристика, соответствующая конденсатору $C_{3}$, лучшим образом подходит для процесса передачи энергии. Основанием для такого заключения является форма этой характеристики с рациональным сочетанием участка с большим статизмом (ограничение тока) с участком малого статизма (ограничение напряжения), которая достаточно близко соответствует алгоритму заряда литий-ионных аккумуляторных батарей. При этом эффект от введения последовательного резонансного конденсатора заключается в увеличении эффективности системы в целом при минимизации массы и габаритов трансформатора.

Выбор конкретного значения емкости $C_{P 2}$ можно обосновать при анализе частотных характеристик вторичной цепи, показанных на рис. 7, где приведены зависимости передаваемой активной мощности $P_{2}^{*}$ и габаритной мощности $S_{C}^{*}$ этого конденсатора в относительных единицах от относительной резонансной частоты тока $n_{2 I}=f / f_{2 P}$ при этом переменные $P_{2}^{*}$ и $S_{C}^{*}$ определяются в отношении к передаваемой мощности без резонансного конденсатора.

Значения переменных $P_{2}^{*}$ и $S_{C}^{*}$ возрастают до бесконечности на частоте резонанса напряжений

$$
f_{2 U}=\frac{1}{2 \pi \sqrt{\left(1 k^{2}\right) L C}},
$$

где $C_{P 2}, L_{2}$ - емкость резонансного конденсатора и собственная индуктивность вторичной обмотки со-

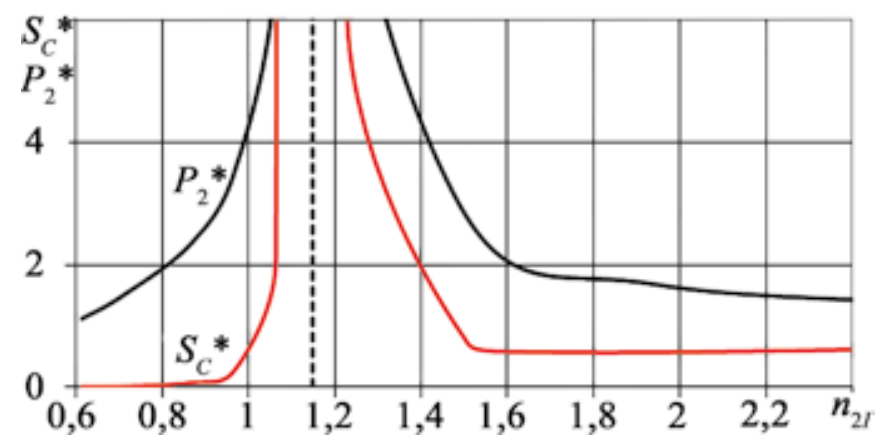

Puc. 7. Частотные характеристики вторичной цепи системы при последовательном включении резонансного конденсатора ответственно; $k$ - коэффициент магнитной связи трансформатора [6].

Частота резонанса токов для вторичной цепи системы:

$$
f_{2 I}=\frac{1}{2 \pi \sqrt{L_{2} C_{P 2}}},
$$

т.е. исходя из (5) и (6) частота $f_{2 I}$ резонанса токов для вторичной цепи системы будет всегда меньше частоты $f_{2 U}$ резонанса напряжений.

По графикам, приведенным на рис. 7, можно установить связь между относительной частотой резонанса токов $n_{2 I}=f / f_{2 l}$, соответствующей активной мощностью $P_{2}^{*}$, передаваемой системой, и габаритной мощностью $S_{C}^{*}$ резонансного конденсатора. Значения этих переменных являются определяющими при решении компромиссной задачи выбора резонансной частоты $n_{2 I}$ при желаемом увеличении за счет резонанса передаваемой активной мощности $P_{2}^{*}$ и приемлемых габаритах резонансного конденсатора, прямо связанных с габаритной (реактивной) мощностью $S_{C}^{*}$.

Так, если принимается решение увеличить передаваемую мощность в 3 раза, то, в соответствии с рис. 7, это потребует применения последовательного резонансного конденсатора с относительной габаритной мощностью примерно $S_{C}^{*} \cong 0,1$. Выбранному режиму соответствует относительная резонансная частота тока $n_{2 I} \cong 0,93$, значение которой, с использованием (6), позволяет определить емкость последовательного резонансного конденсатора $C_{P 2}$.

Очевидно, здесь могут быть приняты и другие решения в зависимости от поставленной задачи и обозначенных приоритетов. Если, например, требуется получить большее увеличение передаваемой мощности, то, используя графики на рис. 7, можно найти соответствующие этой мощности значения относительной частоты резонанса токов и габаритной мощности конденсатора. Последняя в этом случае будет также иметь большее значение.

Важно отметить, что предлагаемый подход к повышению эффективности системы бесконтактного заряда имеет такое достоинство, как возможность независимого воздействия на режимы работы первичной и вторичной сторон трансформатора. Действительно, применяя на первичной стороне резонансную цепь РЦ1 из последовательно соединенных емкости и индуктивности и обеспечивая режим мягкой коммутации транзисторов инвертора, получаем значительное, примерно в 10 раз, снижение мощности потерь на силовых ключах. Добавляя к этому решению резонансную цепь РЦ2 в виде конденсатора, включенного последовательно с вторичной обмоткой 
трансформатора, получаем возможность независимого формирования внешней характеристики системы передачи. Определенное (оптимальное) значение емкости такого конденсатора позволяет снизить массу и габариты трансформатора, что для АНПА является актуальным.

В ряде случаев в качестве резонансной цепи РЦ2 может быть использован конденсатор $C_{P 2}$, включенный параллельно вторичной обмотке трансформатора. При этом резонанс напряжений и резонанс токов на вторичной стороне будут также определяться выражениями (5) и (6) соответственно, а графики внешних характеристик примут вид, как показано на рис. 8 .

Исходная внешняя характеристика системы при $C_{P 2}=0$ имеет при холостом ходе $\left(I_{2}^{*}=0\right)$ ординату, равную единице. Увеличение емкости конденсатора $C_{P 2}$ приводит к увеличению выходного напряжения системы, что иллюстрируют характеристики на рис. 8. При $C_{P 2}=C_{3}$ возникает резонанс напряжений, и ордината внешней характеристики устремляется в бесконечность.

Использование резонансной цепи РЦ2 в виде параллельного конденсатора $C_{P 2}$ может оказаться целесообразным при необходимости иметь повышенное напряжение на вторичной стороне трансформатора. В общем случае достижение этой цели возможно при увеличении числа трансформаторов в системе с включением их вторичных обмоток последовательно, но по условиям компоновки в отсеке АНПА это решение может оказаться проблемным. Выходом может оказаться применение указанной резонансной цепи, что позволит обеспечить требуемый режим с выполнением ограничений по габаритам.

Вместе с тем принимаемое решение должно быть взвешенным и обоснованным, т.к. ограничивая число трансформаторов, необходимо одновременно решать задачу размещения резонансного конденсатора.

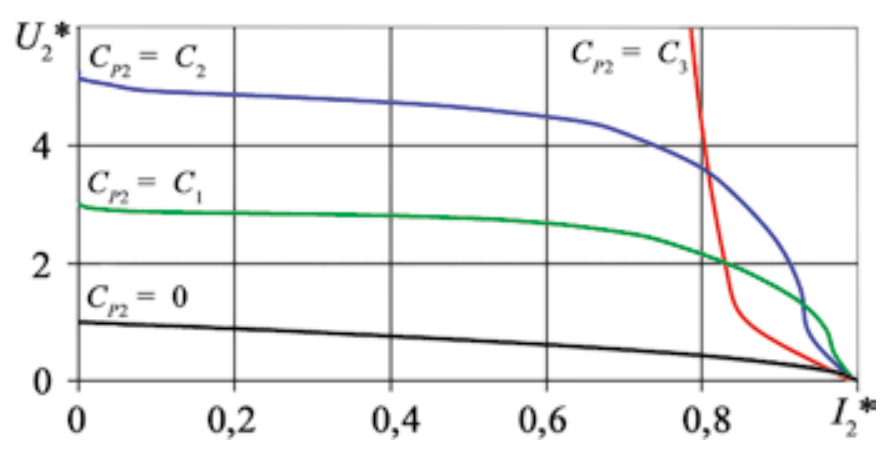

Puc. 8. Внешние характеристики системы передачи при параллельном включении резонансного конденсатора на вторичной стороне трансформатора: $C_{1}<C_{2}<C_{3}$
Выбор конкретного значения емкости параллельного конденсатора $C_{P 2}$ можно выполнить подобно аналогичной задаче для последовательного резонансного конденсатора. Для этого следует рассмотреть частотные характеристики вторичной цепи, показанные на рис. 9, где приведены зависимости передаваемой активной мощности $P_{2}^{*}$ и габаритной мощности $S_{C}^{*}$ этого конденсатора в относительных единицах от относительной резонансной частоты тока $n_{2 I}=f / f_{2 l}$, при этом переменные $P_{2}^{*}$ и $S_{C}^{*}$ определяются в отношении к передаваемой мощности без резонансного конденсатора. Здесь также следует задаваться желаемым увеличением передаваемой активной мощности $P_{2}^{*}$ в отношении к этому же параметру системы без резонанса, при этом определяется относительная резонансная частота тока и далее - габаритная мощность резонансного конденсатора. Найденное значение $n_{2 I}=f / f_{2 I}$ по выражению (6) позволяет определить емкость резонансного конденсатора.

Таким образом, рассмотренные решения, состоящие в использовании резонансных элементов как на первичной, так и на вторичной сторонах трансформатора, сопровождаются суммированием положительных свойств.

Действительно, введение резонансной $L C$-цепи параллельно первичной обмотке трансформатора позволяет уменьшить потери в инверторе, снизить температуру силовых ключей и повысить надежность устройства без влияния на внешнюю характеристику системы. Применение последовательного (или параллельного) резонансного конденсатора на вторичной стороне трансформатора приводит к независимому формированию требуемой внешней характеристики, определяющей передаваемую мощность. При этом, однако, уже будут действовать ограничения, связанные с допустимыми нагрузками на резонансный конденсатор $C_{P}[15]$.

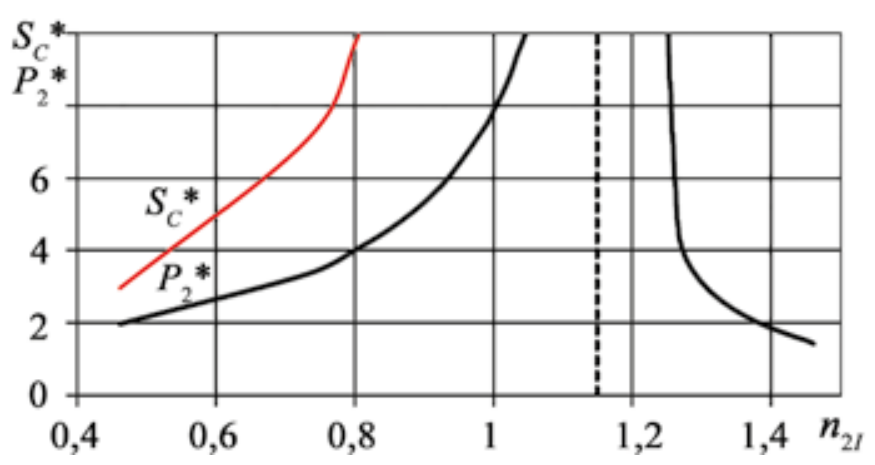

Puc. 9. Частотные характеристики вторичной цепи системы при параллельном включении резонансного конденсатора 


\section{ЗАКЛЮЧЕНИЕ}

Применение предложенных технических решений с использованием параллельной резонансной цепи на первичной стороне трансформатора и резонансного конденсатора на его вторичной стороне по результатам натурного эксперимента в данном частном примере позволило снизить потери в инвертоpe со 130 до 12 Вт при холостом ходе на вторичной стороне трансформатора. В режиме короткого замы- кания на вторичной стороне трансформатора потери в инверторе уменьшаются со 170 до 25 Вт. При этом использование указанных решений позволяет увеличить передаваемую мощность с 212 до 570 Вт. Эти результаты существенно поднимают качественные показатели системы и позволяют заключить, что поставленная задача повышения эффективности системы бесконтактной передачи электроэнергии для заряда АБ подводного аппарата успешно решена.

\section{ЛИТЕРАТУРА}

1. Wang X., Shang J., Luo Z., Tang L., Zhang X., Li. J. Reviews of power systems and environmental energy conversion for unmanned underwater vehicles // Renewable and Sustainable Energy Reviews. 2012. Vol. 16, Is. 4. P. 1958-1970. - URL: http://www.sciencedirect.com/science/article/pii/ S1364032111006095 (дата обращения: 31.01.2020)

2. Valtchev S.S, Baikova S.N., Jorge L.R. Electromagnetic Field as the Wireless Transporter of Energy // Facta Universitatis, Ser: Elec. Energ. 2012. Vol. 25, No. 3. P. 171-181.

3. Singh S.K., Hasarmani T.S., Holmukhe R.M. Wireless Transmission of Electrical Power Overview of Recent Research and Development // Int. Journ. of Computer and Electrical Engineering. 2012. Vol. 4, No. 2, P. 207-211.

4. Пат. 2502170 РФ, МПК Н02Ј3/18. Устройство для бесконтактной передачи электроэнергии на подводный объект (варианты) / Герасимов В.А., Копылов В.В, Кувшинов Г.Е., Наумов Л.А., Филоженко А.Ю., Чепурин П.И. - № 2017138242; заявл.: 03.05.2012; опубл.: 20.12.2013, Бюл. № 35 .

5. Герасимов В.А., Филоженко А.Ю., Чепурин П.И. Структура системы электроснабжения автономного необитаемого подводного аппарата // Изв. ЮФУ. Технические науки. 2013. № 3 (140). С. 47-55.

6. Герасимов В.А., Копылов В.В., Кувшинов Г.Е., Наумов Л.А., Себто Ю.Г. Филоженко А.Ю. Математическая модель устройства для бесконтактной передачи энергии на подводный объект // Подводные исследования и робототехника. 2012. № 2 (14). С. 28-33.

7. Ching-Ming Lai, Ming-Ji Yang, Shih-Kun Liang. A Zero Input Current Ripple ZVS/ZCS Boost Converter with Boumdary-Mode Control // Energies. 2014. Vol. 7, Is. 10. P. 6765-6782.

8. Герасимов В.А., Красковский М.В., Филоженко А.Ю. Автономный инвертор напряжения с последовательным резонансным контуром // Изв. ЮФУ. Технические науки. 2016. № 4. С. 147-158.

9. Пат. 2558681 РФ, МПК Н02М 7/797. Автономный инвертор напряжения для питания нагрузки через трансформатор с низким коэффициентом связи между его обмотками / Кувшинов Г.Е., Наумов Л.А., Себто Ю.Г., Герасимов В.А., Филоженко А.Ю., Чепурин П.И., Красковский М.В. - № 2014111547/07; заявл.: 25.03.2014.; опубл.: 10.08.2015, Бюл. № 22.

10. Герасимов В.А., Красковский М.В, Кувшинов Г.Е., Филоженко А.Ю. Использование резонанса для токовой разгрузки транзисторных ключей инвертора // Двойные технологии. 2016. № 4 (77). С. 55-60.

11. Kraskovskiy M.V., Gerasimov V.A., Kuvshinov G.E., Filozhenko A.Y. The Use of Resonance for Current Downloading of the Transistor Keys of the Inverter // Int. Journ. of Control Theory and Applications. 2016. Vol. 9, Is. 13. P. 305-311.

12. Герасимов В.А., Красковский М.В., Кувшинов Г.Е., Филоженко А.Ю. Повышение эффективности бесконтактной передачи электроэнергии на автономный подводный аппарат // Подводные исследования и робототехника. 2016. № 1 (21). С. 24-30.

13. Пат. 2530877 РФ, МПК Н02J 7/04. Устройство для зарядки аккумуляторной батареи / Герасимов В.А., Филоженко А.Ю., Чепурин П. И.№ 2013113385/07; заявл.: 26.03.2013.; опубл.: 20.10.2014., Бюл. № 29.

14. Пат. 2602078 РФ, МПК Н02Ј 7/02. Устройство для зарядки аккумуляторной батареи подводного объекта / Герасимов В.А., Филоженко А.Ю. - № 2015146625/07; заявл.: 28.10.2015; опубл.: 10.11.2016, Бюл. № 31

15. Film Capacitors. Metallized Polypropylene Film Capacitors (MKP). - URL: http://www.ic-contract.ru/images/pdf/TDK/MKP_B32656S.pdf (дата обращения: 31.01 .2020 ). 